\title{
Adsorption Organic Cationic Dyes of Oxycelluloses and Linters
}

\author{
Michaela Filipi*, Miloslav Milichovský \\ Department of Wood, Pulp and Paper, University of Pardubice, Pardubice, Czech Republic \\ Email: ${ }^{*}$ michaela.filipi@upce.cz
}

Received 19 December 2013; revised 19 January 2014; accepted 26 January 2014

Copyright (C) 2014 by authors and Scientific Research Publishing Inc.

This work is licensed under the Creative Commons Attribution International License (CC BY). http://creativecommons.org/licenses/by/4.0/

(c) (i) Open Access

\begin{abstract}
Natural cellulose and oxycellulose are heterogeneous highly hydrated substances. They have the same chemical composition, but different size of molecules and representation of COOH groups organized into complex supramolecular structure. The oxycellulosic adsorption capacity of organic cationic dyes increases with increasing - $\mathrm{COOH}$ group content and distinctly increases with other competitive ions in the aqueous solution.
\end{abstract}

\section{Keywords}

\section{Oxycellulose; Linters; Adsorption; Cationic Dyes}

\section{Introduction}

\subsection{Basic Dyes}

Basic dyes are cationic soluble salts of coloured bases. This is a class of synthetic dyes, that act as bases and when made soluble in water, they form a colored cationic salt, which can react with the anionic sites on the surface of the substrate. They are applied to substrate with anionic character where electrostatic attractions are formed. The dyes produce bright shades with high tinctorial values, on textile materials and they react on the basic side of the isoelectric points.

Basic dyes are called cationic dyes because the chromophore in basic dye molecules contains a positive charge. They are salts, usually chlorides, in which the dyestuff is the basic or positive radical. Basic dyes are powerful colouring agents. It's applied to wool, silk, cotton and modified acrylic fibres. Usually acetic acid is added to the dyebath to help the take up of the dye onto the fibre. Basic dyes are also used in the coloration of paper, i.e. lignocellulosic materials [1] [2].

*Corresponding author.

How to cite this paper: Filipi, M. and Milichovský, M. (2014) Adsorption Organic Cationic Dyes of Oxycelluloses and Linters. Journal of Encapsulation and Adsorption Sciences, 4, 1-7. http://dx.doi.org/10.4236/jeas.2014.41001 
Waste waters from industries like dye manufacturing, textile dyeing, paper and pupl mills, etc., contain appreciable amounts of basic dyes[3].

\subsection{Chrysoidine R}

Chrysoidine R (Figure 1) is the azo dye with the molecular formula $\mathrm{C}_{13} \mathrm{H}_{14} \mathrm{~N}_{4}$ [4]. It is used to dye silk, cotton, varnish, printing inks, chromed leather, and cellulose-ester plastics. Also used as a biological stain and as a dye in oils, fats, and waxes for polishes. Colour index is 11320 and molecular weight’s $226 \mathrm{~g} / \mathrm{mol}$ [4].

\subsection{Methylene Blue}

Methylene blue (Figure 2) is a heterocyclic aromatic chemical compound with the molecular formula $\mathrm{C}_{16} \mathrm{H}_{18} \mathrm{ClN}_{3} \mathrm{~S}$ and molecular weight: $319 \mathrm{~g} / \mathrm{mol}$. It has many uses in a range of different fields, such as biology and chemistry [1]. At room temperature it appears as a solid, odorless, dark green powder that yields a blue solution when dissolved in water. The hydrated form has 3 molecules of water per molecule of methylene blue [1].

Solutions in water or alcohol have a deep blue color. Methylene blue is used as a dye for a number of different staining procedures [4]. Colour index is 52015 [4].

\section{Description of Experiment}

\subsection{Materials}

As native cellulose was used commercial cotton linter. Oxycelluloses OKCEL H-L were prepared by nitroxide-mediated oxidation of linters and bleached MgBi-sulphite wood pulp inSynthesia, Pardubice-Semtin, Czech Republic—see Table 1 [5].

\subsection{Preparation of Oxycellulose Suspension}

Oxycellulose as well as cotton linters suspension was prepared by weighing out of $20 \mathrm{~g}$ of air dried oxycellulose followed by its dissolution in $1 \mathrm{~L}$ of distilled water. The fibres were stirred up in $1 \mathrm{~L}$ of distilled water. Before stirring up, the oxycelluloses No. 242, No. 247 and linters were refined in order to reach better homogenization of fibrous suspension [5].

Refining of oxycelluloses and linters was carried out in $250 \mathrm{~mL}$ and $500 \mathrm{~mL}$ of distilled water, respectively. The refining of oxycelluloses was carried out in a laboratory blender, using first velocity stage, with duration 4 minutes 30 seconds. The cotton linters refining took place approximately 45 minutes, using second and third velocity stage eventually.

In order to control a sufficient fibres refining, a graduated vessel with the excess of distilled water was used. The small amount of the defibrillated oxycellulose or linters was withdrawn and put into a graduated vessel [5]. The suspension was then shaken up and observed a quality of defibrillation. The refining process was finished in case that no bigger aggregates of fibres were observed in the vessel [5]. As the temperature of the prepared sus-

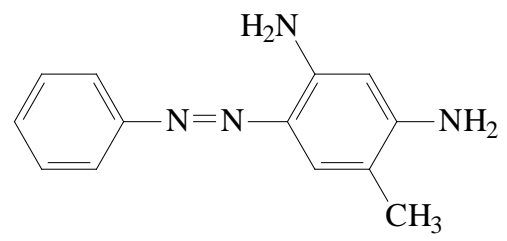

Figure 1. Chrysoidine R.<smiles></smiles>

Figure 2. Methylene blue. 
pension grew up during the refining process, in some cases the suspension was cooled down and kept in the cabinet in order to equilibrate its temperature with the cabinet temperature-at which the measurement was taken place further [5].

As the oxycellulose dissolves in water, it is necessary to keep prepared suspension at least half an hour to reach equilibrium and to avoid further dissolution. Thus, the dissolution changes the consistence of fibrous suspension. Composition, structure and properties of used oxycellulose are summarised in Table 1 [5]. The composition and structure of used oxycelluloses were determined by viscosimetric method and by use of UV-VIS spectroscopy described in literature [6].

\subsection{Methylene Blue (MB)}

The required amount of MB was weighted with precision to 4 decimal places, dissolved in a volumetric flask and made up to volume with distilled water. The prepared solutions were in the concentration range between $6.10^{-4}$ to 2 gMB in $1 \mathrm{~L}$ of distilled water. Prepared solutions with higher concentrations were maintained for at least 30 minutes in a thermostat, that was set to $30^{\circ} \mathrm{C}$. Then the solutions were cooled down to room temperature.

When methylene blue was obtained, the absorption curve was measured. This determination was made for solution with a concentration of $0.01 \mathrm{gMB}$ in $1 \mathrm{~L}$ of distilled water.

\subsection{Chrysoidine R (CHR)}

The preparation of solutions was carried out according to the same method as in methylene blue. The extent of the prepared concentrations was in the range of 0.015 to $0.8 \mathrm{~g}$ CHR in $1 \mathrm{~L}$ of distilled water.

\section{Description of Adsorption Experiments}

While stirring continuously, $50 \mathrm{~mL}$ of oxycellulose suspension was withdrawn and $10 \mathrm{~mL}$ of dyes solution from each calibration solution was added to it [6]. The mixture was intensively stirred up with glass stirrer (300 revolutions per minute) for the period of 10 minutes at the room temperature, e.g. $20^{\circ} \mathrm{C} \pm 1{ }^{\circ} \mathrm{C}$. The prepared suspension was filtered through a fritted glass S2 and dried at the temperature of $70^{\circ} \mathrm{C}$. The amount of ions in the filtrate was then determined by use of spectrophotometry and standard addition method of quantitative analysis approach [7].

The absorbance measurements were always carried out against the filtrate from the blank, which was diluted in the same way as the solutions prepared to measurement by standard addition method. The filtrate blank solution was obtained in the same way as the filtrate dye solution after adsorption with the only difference that in the preparation of the reaction mixture, the suspension was not added to the dye of solution but to pure distilled water.

Determination the equilibrium concentration of dye in the reaction mixture was taken place by filtration of the reaction mixture and by spectrophotometric method of standard additions. This determination must have been

Table 1. Composition, structure and properties of oxycelluloses OKCEL H-L [5].

\begin{tabular}{|c|c|c|c|c|c|c|}
\hline Sample & $\begin{array}{c}\text { Contain of } \\
\text { carboxylic } \\
\text { groups } \boldsymbol{x}_{\mathrm{CoOH}}, \%\end{array}$ & $\begin{array}{c}\text { Degree of } \\
\text { polymerization } \\
\text { DP }\end{array}$ & $\begin{array}{c}\text { Contain of } \\
\text { glucuronic acid } \\
\boldsymbol{x}_{\boldsymbol{G A}}, \mathbf{g} / \mathbf{g}\end{array}$ & $\begin{array}{l}\text { Polydispersion } \\
\text { of } D P \boldsymbol{P}_{\boldsymbol{D P}}\end{array}$ & $\begin{array}{c}\text { Contain of } \mathrm{COOH} \\
\text { groups in PAGA } \\
\boldsymbol{X}_{\boldsymbol{D S}}, \boldsymbol{\%}\end{array}$ & $\begin{array}{l}\text { PAGA destruction-initiating } \\
\text { components GA-PAGA } \\
\text { mmol/g, oxycel }\end{array}$ \\
\hline $205 / 04$ & 19.1 & 20.77 & 0.566 & 1.24 & 67.83 & 0.755 \\
\hline $183 / 05$ & 18.2 & 39 & 0.580 & 1.35 & 58.20 & 0.992 \\
\hline $284 / 05 ; 2 / 3$ & 17.9 & 35.5 & 0.450 & 0.79 & 72.03 & 0.570 \\
\hline $284 / 05 ; 1 / 3$ & 17.8 & 35.8 & 0.456 & 0.81 & 70.84 & 0.651 \\
\hline $242 / 05$ & 11.2 & 59.6 & 0.260 & 0.35 & 59.52 & 0.054 \\
\hline Sulphite & 9.3 & 64.7 & 0.240 & 0.31 & 50.21 & 0.122 \\
\hline $247 / 05$ & 7.5 & 75.4 & 0.287 & 0.40 & 14.61 & 0.053 \\
\hline Linters & 0.1 & 701.3 & 0.048 & 0.05 & 0 & 0.044 \\
\hline
\end{tabular}


made no later than 10 minutes after adsorption, as they began to show flocculation of pigments in the filtered solution and it would interfere with the determinations [8].

\subsection{Equilibrium Concentration of the Dye Adsorbed onto the Pulp Surface $c_{a}$ in g/L}

The determination is performed simply by subtracting the calculated equilibrium concentrations $\boldsymbol{c}_{\text {rovn }}$ from the initial dye concentration in the reaction mixture $\boldsymbol{c}_{\boldsymbol{p}}$ both expressed in $\mathrm{g} / \mathrm{L}$. The initial concentration of the dye in the reaction mixture was calculated from the concentrations of the prepared solutions of the dyes and the volume $(\mathrm{ml})$, which were dosed to the volume of added suspension ( $c_{r . r}$ concentration of dye, $V_{B}$ volume of dye for adsorption, $V_{S}$ volume of suspension for adsorption)

$$
\begin{gathered}
c_{P}=\frac{c_{r . r .} \cdot V_{B}}{V_{B}+V_{S}} \\
c_{a}=c_{p}-c_{\text {rovn }} .
\end{gathered}
$$

\subsection{Equilibrium Concentration of the Dye Adsorbed onto the Pulp Surface $c_{n a a d}$ in $\mathrm{g} / \mathrm{g}$}

This concentration is given in grams of dye per gram of fiber. For its calculation, it is important to know the consistency of the prepared suspension.

The consistency of the suspension can be find be measuring the dry filter solute, which occurs at the end of adsorption. The filter solute was dried by air at room temperature. Weighing was also carried out at room temperature. Assuming constancy the consistency of the oxycellulose suspension after mixing with the dye solution the weight of the filter solute $\boldsymbol{m}_{F K}$ corresponds then to the mass of fibers contained in a 37 or $45 \mathrm{~mL}$ suspension, which are the volumes subscribed to determine $\boldsymbol{V}_{\boldsymbol{S}}$.

$$
x=\frac{1000}{V_{S}} \cdot m_{F K}
$$

Calculation of the absorbed dye concentration in g/g was performed according to the following Formula (4).

$$
c_{\text {naad }}=\frac{c_{a}}{x}
$$

\section{Results and Discussion}

The adsorption isotherms (Figures 3 and 4) shows that the results are highly volatile due to the accompanying colloid-chemical process between adduct dyes with soluble particles oxycellulose which taken place in the supernatant. Followed by filtration of fiber suspension, more or less we will separate these colloidal particles according to their size, affinity for the emerging porosity fibrous bed and porosity influences of other particles that remain in the filtrate, and which we consider as not adsorbed. It is therefore a complex process, where in addition to the sorption process underway and colloidal interactions influenced by dissolved oxycellulose particles, as already mentioned above.

Absence or low initial start-up phase adsorption isotherms so typical in the case of adsorption of $\mathrm{Fe}^{2+}$ ions [5] is probably caused by the aforementioned separation of large colloidal dye adducts with dissolved particles of oxycellulose including cellulose itself. The colloid-chemical process is very complicated because the coagulation of rested hydrocolloid adducts depends on the concentration of the dye, the ratio of dye and coagulation inducing soluble particles of oxycellulose etc. [9]. The process has certain kinetics, which is dependent on mixing. Due to the method of determining the concentration of the not sorbed dye in the supernatant based on its separation of the fiber suspension by filtration, the adsorption isotherm appears as if the surface adsorption proceeded by mechanism of flocculation. It was verified by processing the results obtained using functional dependencies describing the simple Langmuir isotherm accompanied by surface flocculation and adsorption preceded by normal flocculation [10].

From Figures 3 and 4 it can be clearly seen the significant difference between the sorption properties of linters themselves compared to the much higher adsorption capacities of oxycelluloses. There is also a noticeable different cation activity effect of dyes. Methylene blue (highly cationic dye) has a significantly higher sorption 


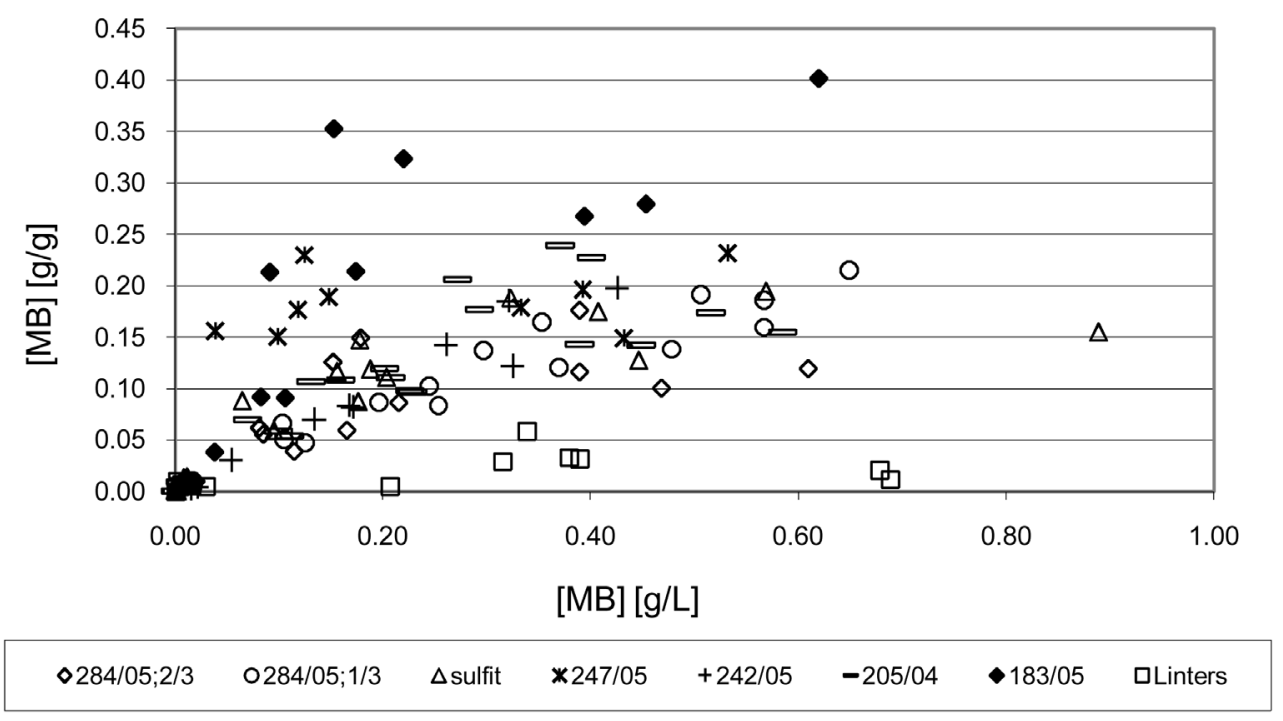

Figure 3. The sorption isotherm of metylen blue on oxycelluloses and cellulose merely in the distilled water. $\mathrm{T}=21^{\circ} \mathrm{C} \pm 1^{\circ} \mathrm{C}$.

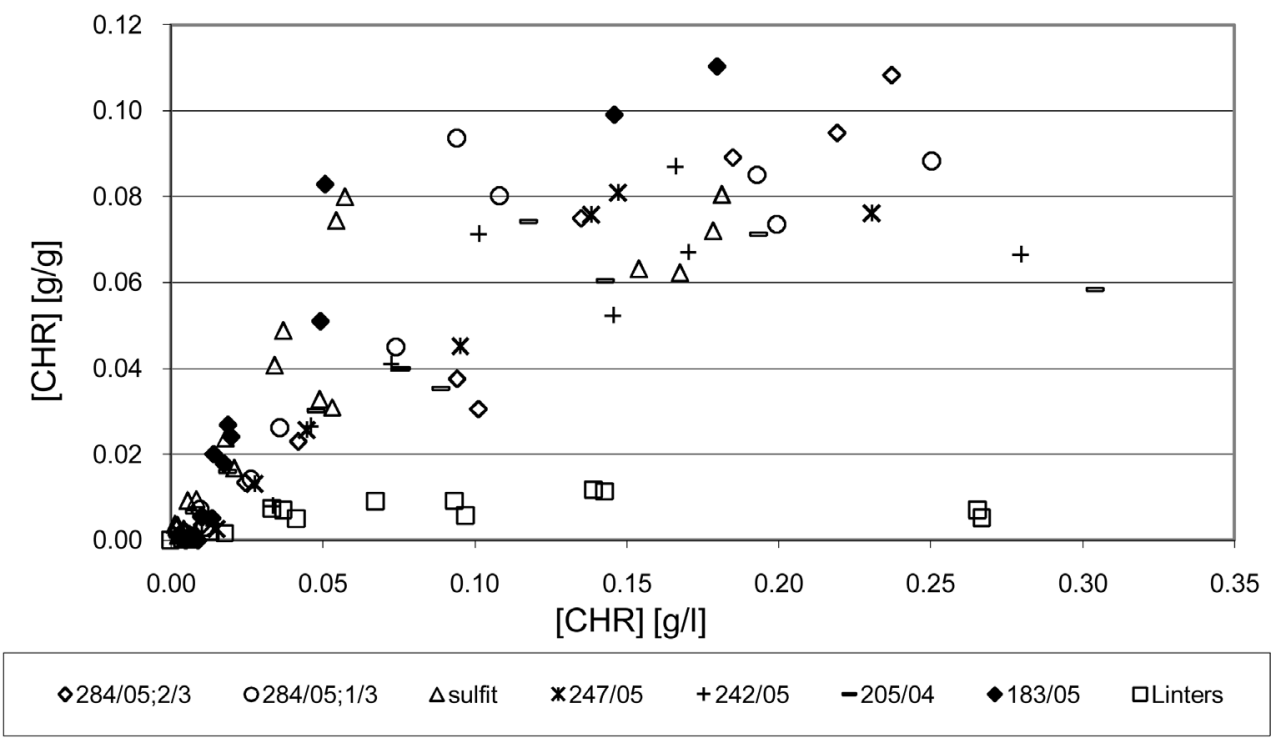

Figure 4. The sorption isotherm of Chrysoidin $\mathrm{R}$ on oxycelluloses and cellulose merely in the distilled water. $\mathrm{T}=21^{\circ} \mathrm{C} \pm 1{ }^{\circ} \mathrm{C}$.

properties to oxycelluloses than Chrysoidine R (weakly cationic). For oxycelluloses the adsorption isotherms differ from each other more in the case of methylene blue (Figure 3) than in the case of Chrysoidine R (see Figure 4). This is probably due to the influence of different cation activity of these dyes. Strongly cationic Methylene blue is more sensitive to the composition and structure of oxycellulose than the less cationic Chrysoidine R.

With considerably best adsorptive properties towards studied dyes, especially due to the methylene blue, appear to be Oxycellulose 183/05, although it does not have the highest content of $\mathrm{COOH}$ groups. It has a high content of free glucuronic acid, also contains the highest amounts of destruction-initiating components GAPAGA, the highest polydispersity of the molecular weight distribution $\boldsymbol{P}_{\boldsymbol{D P}}$ and a relatively high degree of polymerization $\boldsymbol{D P}$ but a relatively low proportion of $\mathrm{COOH}$ groups in PAGA $\boldsymbol{X}_{\boldsymbol{D S}}$ itself. This seeming illogicality in the individual indicators confirms the theory above that the seemingly high adsorption capacity of this oxycellulose to cationic dyes is mainly caused by coagulation of colloidal dye adducts with colloidal particles of 
oxycellulose, with a significant contribution of their free hydrocolloids with a strong anionic nature. Conversely, oxycellulose with the lowest content of $\mathrm{COOH}$ groups, free glucuronic acid $\boldsymbol{x}_{\boldsymbol{G A}}$, polydispersity of the molecular weight distribution $\boldsymbol{P}_{\boldsymbol{D P}}$, representation of $\mathrm{COOH}$ groups in PAGA $\boldsymbol{X}_{\boldsymbol{D S}}$ alone including destruction-initiating components GA-PAGA, but the highest $\boldsymbol{D P}$ represented by oxycellulose 247/05 also features with interesting adsorption properties, although markedly lower adsorption capacity compared with more oxidised oxycellulose. At this oxycellulose it is clearly shown that the mechanism of the course of sorption processes in the case of adsorption of substances with aggregation capabilities is controlled by surface flocculation. It also causes still relatively high adsorption capacity of oxycellulose compared to only cellulose represented in this case with linters. From a practical point of view, in this sense also oxidised MgBi bleached sulphite pulp behaves, which has a similar composition, properties, and absorption behaviour as oxycellulose 247/05. In this case, however, the process of adsorption is by the mechanism of surface flocculation less satisfactory. Probably, due to relatively high solubility of oxycellulose (see Table 1), the adsorption process is strongly influenced by the above mentioned accompanying colloid processes.

\section{Conclusions}

Excellent adsorption properties of oxycellulose to organic cationic dyes were demonstrated. Thanks to the varying composition of oxycellulose, which depends on the structure of PAGA, this behaviour is complicated and the shape of the absorption isotherms can be divided into:

-Adsorption isotherms with a typical sigmoid character with a strong initial plateau characteristic for lowmolecular substances with a low tendency to agglomerate;

-Adsorption isotherms with a slight S-shape typical for so-called surface flocculation and characteristic for substances with a strong tendency to agglomeration or coagulation of the adsorbed molecules or nanoparticles in case of their increased concentration.

It is logical that it must be expected, that if compounds of inorganic and organic nature formed by reaction between dissolved particles of oxycellulose and sorbing ingredients are of a colloidal nature, so therefore their behaviours, e.g. stability will act according to the rules of stability of colloidal systems.

\section{Acknowledgements}

The authors thank the ESF and state budget of Czech Republic for financial support. This research was supported by grants VEPACZ.1.07/2.3.00/20.0236.

\section{References}

[1] Arient, J. (1968) Přehled Barviv. SNTL Prague Czechoslovakia.

[2] Matouš, V. (1987) Chemie Organických Barviv. SNTL Prague Czechoslovakia.

[3] Saliba, R., Gauthier, H., Gauthierand, R. and Petit-Ramel, M. (2002) The Use of Amidooximated Cellulose for the Removal of Metals Ions and Dyes from Waste Water. Cellulose, 9, 183-191. http://dx.doi.org/10.1023/A:1020117032406

[4] (2013) http://pubchem.ncbi.nlm.nih.gov/summary/summary.cgi?cid=6099

[5] Filipi, M. and Milichovský, M. (2008) Adsorption Characteristic of Oxycellulose in a Competitive Environment with Other Substance. Adsorption Science \& Technology, 26, 545-561. http://dx.doi.org/10.1260/0263-6174.26.7.545

[6] Milichovsky, M. and Milichovska, Sv. (2008) Characterization of Oxidized Cellulose by Using UV-VIS Spectroscopy. Journal of Applied Polymer Science, 107, 2045-2052. http://dx.doi.org/10.1002/app.27232

[7] Malát, M. (1973) Absorption Inorganic Photometry. Academia Prague Czechoslovakia.

[8] Klouda, P. (1996) Moderní Analytické Metody. Pavel Klouda Ostrava Czechoslovakia.

[9] Milichovsky, M. and Češek, B. (2002) Surface Flocculation as a New Tool for Controlling Adsorption Processe. Adsorption Science \& Technology, 20, 883-896. http://dx.doi.org/10.1260/02636170260555796

[10] Milichovsky, M., Sopuc, T. and Richter, J. (2007) Depolymerization during Nitroxide-Mediated Oxidation of Native Cellulose. Journal of Applied Polymer Science, 106, 3641-3647. http://dx.doi.org/10.1002/app.24540 


\section{Nomenclatures}

$c_{\text {rovn }}$ : equilibrium concentration of dye $(\mathrm{g} / \mathrm{L})$

$c_{a:}$ equilibrium concentration of the dye adsorbed onto the pulp surface $(\mathrm{g} / \mathrm{L})$

$c_{\text {naad }}$ : equilibrium concentration of the dye adsorbed onto the pulp surface $(\mathrm{g} / \mathrm{g})$

$c_{p}$ : total concentration of the dye in the aqueous system $(\mathrm{g} / \mathrm{L})$

$c_{r . r}$ : concentration of dye $(\mathrm{g} / \mathrm{L})$

$c_{S}$ : concentration of standard solution $(\mathrm{g} / \mathrm{L})$

$M$ : dye

$m_{F K}$ : weight filtration cake (g)

$V_{B}$ : volume of dye for adsorption $(\mathrm{mL})$

$V_{S}$ : volume of suspension for adsorption $(\mathrm{mL})$

$x$ : consistency of suspension $(\mathrm{g} / \mathrm{L})$

$D P$ : degree of polymerization

$P_{D P}$ : number characterizing the polydispersity in oxycellulose

$D S_{P A G A}$ : degree of substitution of PAGA

PAGA: poly(1,4- $\beta$-D anhydroglucuronic acid)

$x_{\mathrm{COOH}}$ : concentration fo carboxyl groups in oxycellulose (\%)

$X_{D S}$ : share of COOH groups in PAGA (\%)

$x_{G A}$ : content of free glucuronic acid in oxycellulose $(\mathrm{w} / \mathrm{w})$ 